\title{
Concentraciones séricas de Beta- hidroxibutirato y su asociación con enfermedades posparto en bovinos de leche
}

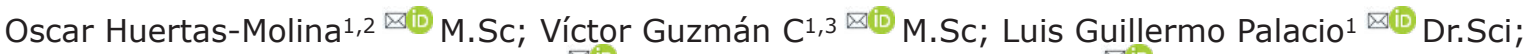 \\ Jorge Zambrano-Varón ${ }^{凶} \otimes \mathbb{D}$ Ph.D; Martha Olivera-Angel ${ }^{*} \bowtie \mathbb{D}$ Dr. Sci. Agr.
}

\begin{abstract}
1 Universidad de Antioquia, Grupo de investigación Biogénesis, Carrera 75 N²65-87, Medellín, Colombia.
${ }^{2}$ Corporación Colombiana de Investigación Agropecuaria - AGROSAVIA. Centro de Investigación El Nus. San José del Nus, Antioquia. ${ }^{3}$ Bayer Animal Health, Carrera 58 No. 10-76, Bogotá, Colombia.

4 Universidad Nacional de Colombia, Grupo de Reproducción Animal y Salud de Hato, Carrera 45 N²6-85, Bogotá, Colombia.

*Correspondencia: martha.olivera@udea.edu.co
\end{abstract}

Recibido: Noviembre 2019; Aceptado: Junio 2020; Publicado: Agosto 2020.

\section{RESUMEN}

Objetivo. Determinar la prevalencia de cetosis bovina según las concentraciones séricas ( $\mathrm{mmol} / \mathrm{Lt}$ ) de beta-hidroxibutirato $(\beta \mathrm{HB})$ y estimar su asociación con enfermedades del posparto temprano en vacas lecheras. Materiales y métodos. Se llevó a cabo un estudio epidemiológico de corte transversal con información individual de los niveles sanguíneos de $\beta \mathrm{HB}$ y presentación clínica de enfermedades puerperales de $n=1149$ animales; además se evaluó la eficiencia de la prueba como predictora de enfermedad posparto. Posteriormente, se realizó $X^{2}$ y un modelo de regresión logística final para explorar los factores asociados con hipercetonemia. La relación entre $\beta H B$ sanguíneo, la presentación de cetosis y la ocurrencia de enfermedades puerperales se calculó analizando Característica Operativa del Receptor (Receiver Operating Characteristic-ROC). Resultados. Según los niveles de $\beta \mathrm{HB}$, la prevalencia de cetosis fue de $7.9 \%$, cetosis clínica $0.6 \%$ y cetosis subclínica $7.3 \%$. La prueba fue predictora de enfermedad puerperal ( $L R+$ del 13.6). La condición corporal $\geq 3.5$, número de partos igual a 2 y $\geq 3$, la presentación de retención de placenta, fiebre de leche y la enfermedad posparto, son factores de riesgo de cetosis. El análisis de la curva ROC mostró que la medición de $\beta H B(\geq 1.2$ $\mathrm{mmol} / \mathrm{Lt})$ en sangre sirve para diagnosticar cetosis $(\mathrm{p}<0.0001)$. Conclusiones. La medición de niveles sanguíneos de $\beta$ HB permitió determinar que la presentación de cetosis es baja, es una alerta no solamente para esta enfermedad sino para las enfermedades del posparto temprano. Los resultados de este estudio confirman lo reportado en estudios previos sobre los factores de riesgo de cetosis.

Palabras clave: Cetosis; factores de riesgo; hipercetonemia; lactancia temprana; Odds Ratio (Fuente: $\mathrm{MeSH}$, National agricultural library).

\section{ABSTRACT}

Objective. Determine the prevalence of bovine ketosis based on serum concentrations (mmol/ $\mathrm{Lt})$ of beta-hydroxybutyrate $(\beta \mathrm{HB})$ and estimate its association with early postpartum diseases in dairy cows. Materials and methods. An epidemiological cross-sectional study was carried out with

Como citar (Vancouver).

Huertas-Molina O, Guzman CV, Palacio BL, Zambrano-Varón J, Olivera-Angel M. Concentraciones séricas de beta-hidroxibutirato y su asociación con enfermedades posparto en bovinos de leche. Rev MVZ Córdoba. 2020; 25(3):e1821. https://doi.org/10.21897/rmvz.1821 
individual information on blood levels of $\beta \mathrm{HB}$ and clinical presentation of puerperal diseases in 1149 animals; In addition, the efficiency of the test was evaluated as a predictor of postpartum disease. Subsequently, univariate analysis and a final logistic regression model were performed to explore the factors associated with hyperketonemia. The association between blood $\beta \mathrm{HB}$, the presentation of ketosis and the occurrence of puerperal diseases was calculated by analyzing the Receiver Operating Characteristic -ROC. Results. According to $\beta H B$ levels, the prevalence of ketosis was $7.9 \%$, clinical ketosis $0.6 \%$ and subclinical ketosis $7.3 \%$. The test was a predictor of puerperal disease (LR+ of 13.6). Body condition score $\geq 3.5$, number of births 2 and $\geq 3$, presentation of retained fetal membranes, milk fever and postpartum disease, are risk factors for ketosis. The analysis of the ROC curve showed that the measurement of $\beta \mathrm{HB}(\geq 1.2 \mathrm{mmol} / \mathrm{Lt})$ in blood serves to diagnose ketosis $(p<0.0001)$. Conclusions. The measurement of $\beta \mathrm{HB}$ blood levels allowed to determine that ketosis presentation is low, it is an alert not only for this disease but for early postpartum diseases. The results of this study confirm risk factors observed in previous studies.

Keywords: Ketosis; risk factor; hyperketonemia; early lactation; Odds Ratio (Fuente: MeSH, National agricultural library).

\section{INTRODUCCIÓN}

La cetosis es un desorden metabólico en el cual aumentan los niveles circulantes de cuerpos cetónicos (acetona, acetoacetato y $\beta \mathrm{HB}$ ) como consecuencia del incremento de AGNE (Ácidos Grasos No esterificados) circulantes o de los requerimientos energéticos, estos últimos a su vez pueden superar la capacidad de ingesta de carbohidratos durante el posparto temprano y resultar en una falla de los mecanismos de adaptación al balance energético negativo (BEN), lo que conduce a hipercetonemia y la presentación de cetosis clínica y subclínica (1) La cetosis es una enfermedad ampliamente distribuida en el mundo. En Europa la prevalencia varía entre $4.9 \%$ y $72 \%$, en Norte América se ha reportado entre el $12.1 \%$ y $61 \%$ y en América hasta el $21 \%$ (2). Dos estudios en Colombia reportaron una prevalencia de 8.3 y $48.6 \%(3,4)$. Como se puede observar, en muchas partes del mundo y en Colombia el rango de prevalencia reportado puede deberse a la distribución heterogénea de la población.

Los hallazgos paraclínicos más representativos son la hipoglicemia y la alta concentración de cuerpos cetónicos (hipercetonemia) en sangre, orina, leche (5) y tejidos extrahepáticos $(6,7)$. Acorde con la revisión de la epidemiología de la cetosis (7), esta enfermedad se ha clasificado en dos tipos según su origen. La cetosis Tipo I, que ocurre cuando los requerimientos energéticos del animal superan la capacidad gluconeogénica de los hepatocitos, por falta de oxalacetato y propionato e incremento de actividad de la enzima Carinitil Palmitoil Transferasa 1 (CPT1) (6). La cetosis Tipo II se ha relacionado con sobrecondicionamiento energético durante el periodo seco (8) y la inapetencia causada por la presentación de enfermedades del posparto temprano, principalmente metritis y mastitis (7). Los animales con este tipo de cetosis tienen comprometida la función inmune por el incremento agudo de AGNE circulantes, citoquinas, adipoquinas y proteínas de fase aguda (9).

La forma más conveniente de clasificar la enfermedad es acorde con la presentación de signos clínicos $(7,10)$. La cetosis clínica cursa con disminución progresiva del consumo, pérdida de peso marcada, deshidratación, heces secas, pica y disminución en la producción de leche (7). Casos clínicos severos presentan una sintomatología nerviosa como lameteo constante, convulsiones, aparente ceguera y ataxia (10). Estos signos se han relacionado adicionalmente, con niveles de $\beta H B$ sanguíneo y lácteo de $\geq 3 \mathrm{mmol} /$ Lt $y \geq 0.2$ $\mathrm{mmol} / \mathrm{Lt}$, respectivamente (1).

La cetosis subclínica se identifica por la detección de hipercetonemia en sangre, leche y orina y sin la presentación de cuadros clínicos. Las concentraciones plasmáticas de $\beta \mathrm{HB}$ compatibles con los efectos negativos sobre la producción y la salud son $\geq 1.2$ y $\leq 2.9 \mathrm{mmol} / \mathrm{Lt}(1,11)$.

Estudios recientes han definido con cetosis a todo aquel animal que presente concentraciones séricas de $\beta H B \geq$ a $1.2 \mathrm{mmol} / \mathrm{Lt}(3,4)$.

Se han descrito distintos factores asociados con el riesgo de presentación de cetosis en sistemas productivos bajo condiciones de estabulación y pastoreo $(2,4,5,12,13,14)$. Estudios han reportado como factores de riesgo para la ocurrencia de cetosis la raza,(OR:1.46, 
IC 95\% (1.23 - 1.73) p<0.05) (12), el número de partos (tres o más partos) (OR: 2.8, IC $95 \%(2.0$ - 3.7), p<0.01) (2), el alto puntaje de condición corporal (CC) (OR: 5.25, IC 95\% $(1.32-21.11), p=0.0188)(15)$, la presentación de enfermedades puerperales tales como la mastitis (OR: 1.9, IC 95\% (1.3-2.7), p<0.01) (16), el desplazamiento abomasal (OR: 24.6, IC 95\% (5.65-107.4), p<0.0001) (5), la retención de membranas fetales (OR: 1.6, IC 95\% (1.1 $2.3), p=0.01)(16)$, claudicación (OR: 2.1, IC $95 \%(1.5-2.7), \mathrm{p}<0.0001)(13)$ y la metritis (OR: 4.94, IC 95\% (1.17-0.98), $p=0.0302$ ) (15).

Desde el punto de vista fisiológico, la hipercetonemia también se puede considerar como un factor predisponente para la ocurrencia de cetosis clínica (OR: 14.7 (IC 95\% (7.2-29.8) $\mathrm{p}<0.01)$ ) y enfermedades puerperales (1) como desplazamiento de abomaso; desencadenada por signos clínicos de cetosis como inapetencia, disminución o ausencia de la movilidad ruminal (17). Por otro lado, el incremento patológico de los niveles circulantes de $\beta \mathrm{HB}$ puede favorecer la presentación de metritis (18) y claudicación (19), por la correlación de esta enfermedad con estrés oxidativo y disminución de la función inmune (19). Además de las enfermedades concomitantes y los signos clínicos de la cetosis; la cetosis subclínica genera pérdidas desapercibidas de producción de leche y disminución de consumo (4), lo que resulta en incremento de los costos de producción (1). En Colombia, se han hecho pocos estudios sobre la prevalencia y las consecuencias de la cetosis sobre la producción de leche.

Este es un estudio epidemiológico de corte transversal, su objetivo consistió en establecer la prevalencia de cetosis según los niveles de $\beta \mathrm{HB}$ en los departamentos de Antioquia, Boyacá, Caldas y Cundinamarca y su asociación con la ocurrencia de enfermedades puerperales en bovinos de leche durante el posparto temprano bajo condiciones de pastoreo.

\section{MATERIALES Y MÉTODOS}

Base de datos. Para este estudio se usó la base de datos suministrada por Bayer S.A para que se hicieran los análisis respectivos acorde con la información registrada en esta: Departamento muestreado, número de predios muestreados, número de vacas por predio, vaca parida entre 7 y 15 días posparto, en la base de datos se registró, además el nivel de $\beta \mathrm{HB}$ sérico y la presentación de las enfermedades listadas en la Tabla 1.

Tabla 1. Definición de enfermedades posparto según Brunner et al (3).

\begin{tabular}{|c|c|}
\hline Enfermedad & Definición \\
\hline $\begin{array}{l}\text { Retención de } \\
\text { placenta }\end{array}$ & $\begin{array}{l}\text { Falla en la expulsión de membranas fetales } \\
\text { mayor a } 24 \text { horas. }\end{array}$ \\
\hline Metritis & $\begin{array}{l}\text { Aumento de tamaño del útero con/sin } \\
\text { secreción uterina purulenta y de mal } \\
\text { olor asociada con signos sistémicos } \\
\text { (Fiebre, inapetencia/anorexia, inactividad, } \\
\text { disminución de la producción de leche). }\end{array}$ \\
\hline Mastitis & $\begin{array}{l}\text { Leche visiblemente anormal con o sin } \\
\text { cambios en la apariencia de la ubre (signos de } \\
\text { inflamación, enrojecimiento o cuartos duros). }\end{array}$ \\
\hline $\begin{array}{l}\text { Fiebre de } \\
\text { Leche }\end{array}$ & $\begin{array}{l}\text { Vaca de } 24 \text { - } 48 \text { horas posparto que requiere } \\
\text { inyección de Calcio debido a signos clínicos } \\
\text { de hipocalcemia, como debilidad muscular } \\
\text { y decúbito patológico postparto. }\end{array}$ \\
\hline $\begin{array}{c}\text { Desplazamiento } \\
\text { de abomaso }\end{array}$ & $\begin{array}{l}\text { Distensión y desplazamiento del abomaso } \\
\text { hacia la derecha o la izquierda. Presencia del } \\
\text { sonido de "ping" a la auscultación y percusión } \\
\text { abdominal y confirmado por cirugía. }\end{array}$ \\
\hline Claudicación & $\begin{array}{l}\text { Presentación de claudicación con puntaje } \\
\text { de locomoción } \geq 3 \text { (escala de } 1-5 \text { ) y con } \\
\text { hallazgos anormales durante el examen } \\
\text { de la pezuña (como dermatitis digital e } \\
\text { interdigital, enfermedad de la línea blanca, } \\
\text { absceso de la suela y trauma, entre otros). }\end{array}$ \\
\hline Cetosis clínica & $\begin{array}{l}\text { Disminución de la producción de leche, } \\
\text { consumo e inapetencia, actividad reducida, } \\
\text { prueba positiva ( } \beta \mathrm{HB} \geq 1.2 \mathrm{mmol} / \mathrm{Lt}) \mathrm{de} \\
\text { cuerpos cetónicos en sangre o leche, } \\
\text { ausencia de desplazamiento de abomaso u } \\
\text { otras causas primarias, olor a acetona en } \\
\text { el aliento o leche }\end{array}$ \\
\hline
\end{tabular}

El análisis previo mostró que el número de predios fue: 52 en Antioquia, 5 en Boyacá, 3 en Caldas y 50 en Cundinamarca, para un total de 1149 animales distribuidos en 40 municipios, cuyos propietarios manifestaron su disponibilidad de participar voluntariamente en el estudio en Antioquia $(n=52)$, Boyacá $(n=5)$, Caldas $(n=3)$ y Cundinamarca $(n=50)$ distribuidos en 40 municipios. El número de animales muestreados por hato osciló entre 2 y 43 y se realizó a lo largo del año 2014. De cada uno de los hatos se obtuvieron muestras de suero sanguíneo de 
entre 2 y 43 animales. El $92.5 \%$ de los animales de este estudio fueron de la raza Holstein, el porcentaje restante estuvo compuesto por otras razas lecheras.

Aspectos éticos. Para el procesamiento de los datos se firmó un acta entre la empresa Bayer S.A y la Universidad de Antioquia en la que se autorizó el uso de la información para divulgar y discutir los resultados obtenidos del estudio.

Obtención de muestras de sangre y medición de $\boldsymbol{\beta H B}$. A partir de una muestra sanguínea proveniente de la vena coccígea, se determinó inmediatamente la concentración sérica de $\beta H B$, mediante el uso de un medidor portátil (Precision Xceed, Abbott Diabetes Care Inc., Alameda, CA), que previamente fue validado para el uso en bovinos (20). Cuando se trabaja con un límite superior de $1.2 \mathrm{mmol} \beta \mathrm{HB} / \mathrm{Lt}$, la sensibilidad y especificidad de la prueba oscila entre $75-96.3 \%$ y $91.0-98.0 \%$, respectivamente (4).

Definición de las variables. La unidad de estudio fue el animal. Cetosis se definió según Tatone et al (20), a toda muestra con una concentración $\geq 1.2 \mathrm{mmol} / \mathrm{Lt}$ de $\beta \mathrm{HB}$. Se utilizaron variables independientes categóricas clasificadas de la siguiente manera: 1) número de partos $(1,2, y \geq 3), 2)$ condición corporal $(\leq 2.75,>2.75-<3.5$ y $\geq 3.5)$, 3) el día posparto (7-15) en el que se obtuvo la muestra, 4) presentación de enfermedades del posparto ( $\mathrm{Si}$ / No) y 5) enfermedad posparto definida como el conjunto de la presentación de una o más enfermedades.

Las enfermedades posparto fueron diagnosticadas mediante examen clínico por médicos veterinarios y acorde con las definiciones establecidas en la tabla 1.

Análisis Estadístico. La prevalencia general de cetosis subclínica se estimó cuando las concentraciones séricas de $\beta H B$ fueron $\geq 1.2-<3 \mathrm{mmol} / \mathrm{Lt}$ ) (13) y de cetosis clínica cuando la concentración fue $\geq 3 \mathrm{mmol} / \mathrm{Lt}$ (2). Adicionalmente, se calculó la prevalencia de enfermedades puerperales el mismo día de la toma de sangre y teniendo en cuenta el registro de la base de datos tomada por los veterinarios, así, se estimó su asociación con las concentraciones séricas de $\beta \mathrm{HB} \geq 1.2 \mathrm{mmol} /$ Lt. Este último fue el punto de corte para definir cetosis (13) Además, se evaluó la eficiencia de la prueba como predictora de cada una de las enfermedades y las enfermedades en conjunto. Para cumplir con este objetivo se calcularon:

a. Prevalencia verdadera (PV): proporción de animales que presentaron cada una de las enfermedades puerperales, la población riesgo de este cálculo son todos los animales incluidos en el estudio (21).

b. Prevalencia aparente (PA): proporción de animales positivos a cetosis (Población riesgo) y a cada una de las enfermedades puerperales (21). c. Sensibilidad: probabilidad condicional de que un animal detectado con cetosis por la prueba ( $\beta \mathrm{HB} \geq 1.2 \mathrm{mmol} / \mathrm{Lt}$ ) corresponda a un caso diagnosticado de enfermedad puerperal (21).

d. Especificidad: probabilidad condicional de que un animal sea negativo para cetosis bovina $(\beta \mathrm{HB}<1.2 \mathrm{mmol} / \mathrm{Lt}$ en aquellos animales que no sufrieron una enfermedad puerperal (21).

e. Valor predictivo positivo (VPP): proporción de animales que fueron identificados con cetosis por la prueba y que realmente presentaron una enfermedad posparto (21).

f. Valor predictivo negativo (VPN): proporción de animales que siendo identificados como negativos para cetosis por la prueba, realmente no presentaron enfermedad posparto (21).

g. Indice de probabilidad (IP) o Likelihood Ratio (LR) positivo: expresa la posibilidad de observar un resultado positivo, mediante un contraste entre los animales con y sin la enfermedad en cuestión (Enfermedad puerperal). Para el caso de este estudio, LR positivo significa el incremento en la probabilidad de presentar enfermedad puerperal cuando el animal presentó concentraciones de $\beta \mathrm{HB}>1.2$ $\mathrm{mmol} / \mathrm{Lt}$, en comparación a aquellos positivos a cetosis sin enfermedad puerperal.

Según McGee (22) se puede interpretar que en los casos en que LR es 2-5, las concentraciones de $\beta H B \geq 1.2 \mathrm{mmol} / \mathrm{Lt}$ no implican que la prueba identifique animales con alguna condición patológica postparto mientras que dicha probabilidad mejora su predicción moderadamente cuando el LR $\geq 5-<10$. Cuando el LR es >10, la prueba es muy buena en su capacidad de identificar un animal con alguna condición patológica postparto (22).

Modelos de regresión. Para analizar la asociación entre la prevalencia de cetosis según las concentraciones de $\beta \mathrm{HB}$ y la presentación de enfermedades puerperales se realizó inicialmente un análisis univariado con tablas de contingencia de $2 \times 2$, donde se estimó el Odd Ratio (OR) e 
Intervalo de confianza (IC) del 95\%; se realizó la prueba $X^{2}$, siguiendo los criterios de Hosmer $\&$ Lemeshow y aquellas variables cuyo valor $p<0.2$, se incluyeron en un modelo final de regresión logística binaria. EI OR e IC 95\% calculados permitieron estimar la magnitud de la asociación de las variables que resultaron estadísticamente significativas $(p<0.05)$. Para el análisis estadístico se usó SPSS (IBM Corp. Released 2013. IBM SPSS Statistics for Windows, Version 22.0. Armonk, NY: IBM Corp.).

Para evaluar la relación entre los resultados de la concentración de $\beta \mathrm{HB}$ ( $\mathrm{mmol} / \mathrm{Lt})$, con la presentación de hipercetonemia y la ocurrencia de enfermedades puerperales, se utilizaron curvas ROC. Estas permiten diferenciar la utilidad de la prueba como diagnóstico para hipercetonemia o alguna de las enfermedades puerperales en estudio. Los resultados obtenidos de esta prueba se interpretaron a partir de la medición del área bajo la curva $(A B C)$ y el nivel de significancia de la curva asintótica $(p<0.05)$. Para saber si la medición de $\beta \mathrm{HB}$ sanguíneo puede estár relacionada con hipercetonemia o alguna de las enfermedades puerperales en estudio, se tuvo en cuenta que: Sí el $\mathrm{ABC}=0.5$, la prueba se considera no informativa; si $0.5<A B C$ $\leq 0.7$, exacta; si $0.7<A B C \leq 0.9$, muy precisa; si $0.9<A B C<1$, altamente precisa; y si $A B C=1$, se considera perfecta (13).

\section{RESULTADOS}

A través de la medición de la concentración sérica de $\beta \mathrm{HB}$ de animales provenientes de diferentes sistemas de producción de leche y en condiciones de pastoreo se estimó la prevalencia de cetosis bovina durante el postparto temprano. Según los niveles séricos, la prevalencia de cetosis bovina fue del $7.9 \%$, y osciló entre los días posparto 7-15 (Variación diaria) de 4.4 a 12.5\%. El 0.6\% de la población presentó cetosis clínica y el $7.3 \%$ cetosis subclínica (Figura 1). La mediana de la concentración de $\beta \mathrm{HB}$ fue de $0.6 \mathrm{mmol} / \mathrm{Lt}$ y el rango de variación observado fue entre 0.1-4.2 $\mathrm{mmol} / \mathrm{Lt}$.

El $2.8 \%$ de los animales de primer parto, el $8.7 \%$ de segundo parto y el $10 \%$ de tres o más partos presentaron niveles de $\beta \mathrm{HB} \geq 1.2 \mathrm{mmol} / \mathrm{Lt}$, al igual que el $6.5 \%$ de los animales que tenía un puntaje de CC menor a $2.75,8.7 \%$ con CC entre $2.75-<3.5$ y $17.1 \%$ con CC mayor a 3.5 . El tamaño del hato estuvo entre 28 y 650 animales/ finca donde $46 \%$ tenían menos de 150 animales y el $54 \%, 150$ o más con una prevalencia de cetosis de 8.1 y $7.8 \%$, respectivamente.

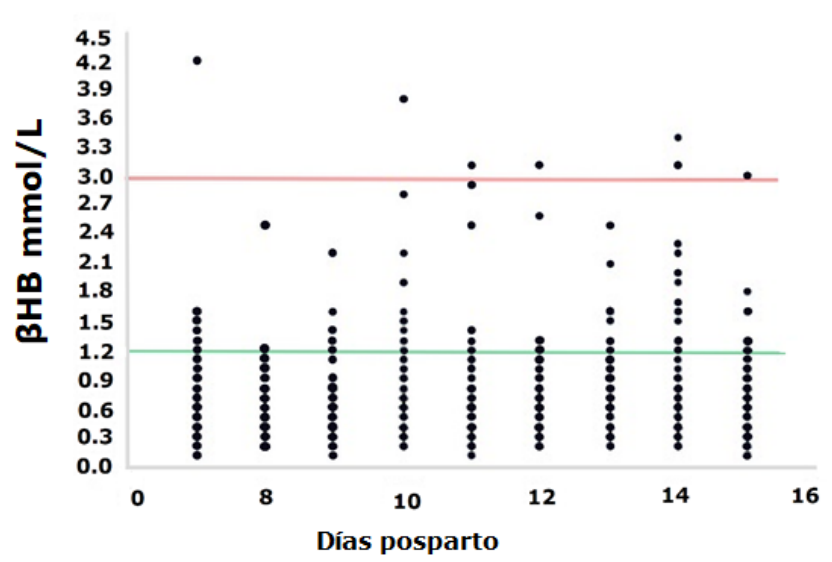

Figura 1. Distribución de concentraciones séricas de $\beta$ HB correspondientes a la presentación de cetosis en bovinos lecheros durante el postparto temprano $(n=1149)$. Cetosis clínica: $\beta H B 3.0 \mathrm{mmol} /$ Lt (Línea Roja); Cetosis subclínica: $\beta \mathrm{HB} \geq 1.2-<3.0 \mathrm{mmol} /$ Lt (Línea verde).

Llama la atención el hecho de que dentro de las enfermedades puerperales evaluadas no se reportaron casos de cetosis clínica. En contraste, $11.1 \%$ de los animales tuvieron un diagnóstico de al menos una enfermedad puerperal cuya prevalencia se distribuyó de la siguiente manera: Mastitis $(4.5 \%)$, retención de placenta $(4.2 \%)$, claudicación $(1.3 \%)$, fiebre de leche $(1.2 \%)$, metritis $(0.8 \%)$ y un caso de desplazamiento de abomaso (0.08\%).

La Tabla 2 expone la evaluación de eficiencia del punto de corte de cetosis ( $\geq 1.2 \mathrm{mmol} / \mathrm{Lt}$ ) para definir la prevalencia de enfermedades puerperales.

Las variables candidatas para entrar al modelo final de Regresión logística asociadas con el aumento de la concentración sérica de $\beta \mathrm{HB}(>1.2$ $\mathrm{mmol} / \mathrm{Lt}$ ) y la presentación de enfermedades puerperales en bovinos de leche fueron: condición corporal, número de partos, retención de placenta, fiebre de leche y enfermedad puerperal. Los resultados del modelo final se muestran en la Tabla 3. 
Tabla 2. Prevalencia de enfermedades postparto en bovinos de leche definida por el incremento de los niveles séricos de $\beta \mathrm{HB}$ ( $\geq 1.2 \mathrm{mmol} / \mathrm{Lt}$ ) y evaluación de la eficiencia de la prueba de diagnóstico.

\begin{tabular}{lcccccccc}
\hline & PV( \%) & PA(\%) & Sen(\%) & Esp(\%) & VPP(\%) & VPN(\%) & IP+ & IP- \\
\hline Fiebre de leche & 1.2 & 0.6 & 50 & 92.6 & 7.7 & 99.3 & 6.3 & 0.5 \\
Retención de placenta & 4.2 & 0.3 & 8.3 & 92.1 & 4.4 & 95.8 & 10.5 & 0.2 \\
Mastitis & 4.5 & 0.1 & 1.9 & 91.8 & 1.1 & 95.2 & 2.3 & 0.9 \\
Desplazamiento de abomaso & 0.08 & 0 & 0 & 92.1 & 0 & 99.9 & 0.0 & 1.1 \\
Claudicación & 1.3 & 0 & 0 & 91.9 & 0 & 98.6 & 0.0 & 1.1 \\
Metritis & 0.8 & 0.2 & 22.2 & 92.2 & 2.2 & 99.3 & 2.8 & 0.8 \\
Enfermedad posparto & 11.1 & 1.1 & 89.8 & 93.4 & 59.4 & 98.6 & 13.6 & 0.1 \\
\hline
\end{tabular}

PV: Prevalencia verdadera; PA: Prevalencia aparente; Se: Sensibilidad; Esp: Especificidad; VPP: Valor predictivo positivo; VPN: Valor predictivo negativo; IP+: Índice de probabilidad positivo (IP+); y negativo (IP-).

Tabla 3. Factores asociados a la ocurrencia del aumento de la concentración sérica de $\beta \mathrm{HB}$ ( $>1.2 \mathrm{mmol} / \mathrm{Lt}$ ) y la presentación de enfermedades puerperales en bovinos de leche.

\begin{tabular}{|c|c|c|c|c|c|c|}
\hline \multirow{2}{*}{ Variable } & \multirow{2}{*}{ Categorías } & \multirow{2}{*}{$\mathbf{n}$} & \multirow{2}{*}{ OR* } & \multicolumn{2}{|c|}{ IC $95 \% * *$} & \multirow{2}{*}{ Valor $\mathrm{p} * * *$} \\
\hline & & & & inferior & Superior & \\
\hline \multirow[t]{3}{*}{ Condición corporal } & $<2.5$ & 260 & & & & \\
\hline & $>2.5-<3.5$ & 563 & 1.2 & 0.7 & 2.1 & 0.51 \\
\hline & $\geq 3.5$ & 76 & 2.7 & 1.3 & 5.9 & 0.011 \\
\hline \multirow[t]{3}{*}{ Número de partos } & 1 & 287 & & & & \\
\hline & 2 & 241 & 3.1 & 1.3 & 7.1 & 0.009 \\
\hline & $\geq 3$ & 621 & 3.7 & 1.7 & 7.8 & 0.001 \\
\hline \multirow[t]{2}{*}{ Desplazamiento de abomaso } & No & 1148 & & & & \\
\hline & $\mathrm{Si}$ & 1 & 0.00 & 0.00 & 0 & 0.9 \\
\hline \multirow[t]{2}{*}{ Mastitis } & No & 1097 & & & & \\
\hline & $\mathrm{Si}$ & 52 & 4.5 & 0.62 & 33.4 & 0.135 \\
\hline \multirow[t]{2}{*}{ Claudicación } & No & 1134 & & & & \\
\hline & $\mathrm{Si}$ & 15 & 3.3 & 0.74 & 14.7 & 0.118 \\
\hline \multirow[t]{2}{*}{ Retención de placenta } & No & 1101 & & & & \\
\hline & Si & 48 & 7.9 & 1.90 & 32.4 & 0.004 \\
\hline \multirow[t]{2}{*}{ Fiebre de leche } & No & 1135 & & & & \\
\hline & $\mathrm{Si}$ & 14 & 11.6 & 3.79 & 35.40 & 0.0001 \\
\hline \multirow[t]{2}{*}{ Enfermedad posparto**** } & No & 1022 & & & & \\
\hline & $\mathrm{Si}$ & 127 & 8.9 & 3.336 & 24.244 & 0.0001 \\
\hline
\end{tabular}

$* \mathrm{OR}=$ Odds ratio

$* *$ IC $95 \%=$ Intervalo de confianza de $95 \%$

$* * *$ Valores $\mathrm{p}<0.05$ son estadísticamente significativos

$* * * *$ Enfermedad Posparto= presentación de al menos una enfermedad.

La curva ROC y el ABC (Área Bajo la Curva) de los resultados de las concentraciones de $\beta H B \geq 1.2$ $\mathrm{mmol} / \mathrm{Lt}$ para cada una de las enfermedades puerperales observadas están resumidas en la Figura 2 y en la Tabla 4.
Se pudo observar (Figura 2 y Tabla 4) que la única entidad asociada con las concentraciones séricas elevadas ( $\beta \mathrm{HB} \geq 1.2 \mathrm{mmol} / \mathrm{Lt}$ ) fue hipercetonemia $(A B C=0.971$; Significancia asintótica $=p<0.001)$. Esto quiere decir que las concentraciones de BHB no están directamente relacionadas con la presentación de enfermedades puerperales. 
Tabla 4. Distribución del Área Bajo la Curva de los resultados de la medición de $\beta H B$ a un punto de corte $\geq 1.2$ $\mathrm{mmol} / \mathrm{Lt}$ en relación con la identificación de patologías en el puerperio temprano en bovinos de leche.

\begin{tabular}{|c|c|c|c|c|c|}
\hline \multirow{3}{*}{ Resultado de la prueba } & \multicolumn{3}{|c|}{ Área bajo la curva } & & \\
\hline & \multirow{2}{*}{ Área } & \multirow{2}{*}{ Error estándar ${ }^{a}$} & \multirow{2}{*}{$\begin{array}{l}\text { Significancia } \\
\text { asintóticab }\end{array}$} & \multicolumn{2}{|c|}{$\begin{array}{c}\text { Intervalo de confianza } 95 \% \\
\text { asintótico }\end{array}$} \\
\hline & & & & Inferior & Superior \\
\hline Hipercetonemia & 0.971 & 0.005 & 0.001 & 0.961 & 0.982 \\
\hline Fiebre de Leche & 0.494 & 0.056 & 0.912 & 0.385 & 0.603 \\
\hline Retención de placenta & 0.479 & 0.054 & 0.704 & 0.373 & 0.584 \\
\hline Mastitis & 0.477 & 0.054 & 0.680 & 0.371 & 0.582 \\
\hline Claudicación & 0.493 & 0.056 & 0.905 & 0.384 & 0.602 \\
\hline Metritis & 0.515 & 0.058 & 0.790 & 0.402 & 0.628 \\
\hline
\end{tabular}

aajo la suposición no paramétrica

bHipótesis nula: área verdadera $=0.5, \mathrm{p}<0.05$ es estadísticamente significativo

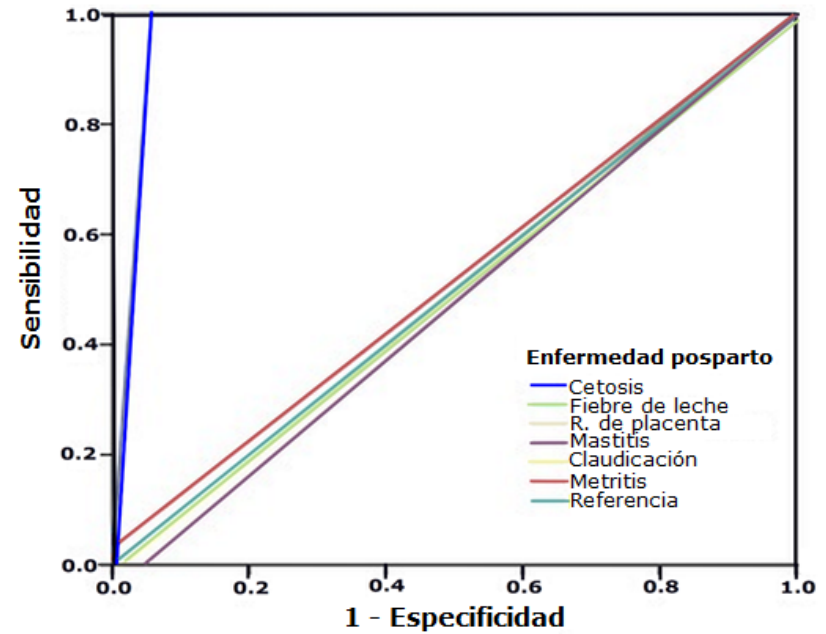

Figura 2. Curva ROC para evaluar la relación entre la medición de la concentración de $\beta \mathrm{HB}$ (mmol/ Lt), la presentación de hipercetonemia y la ocurrencia de enfermedades puerperales en bovinos le leche.

\section{DISCUSIÓN}

En el presente estudio, la prevalencia de cetosis (7.9\%) fue menor a la reportada México $(29.1 \%)$ (23) durante el periodo de 7-15 días posparto. El mismo comportamiento se observó al comparar con estudios que se realizaron durante los primeros 21 días posparto en Colombia ( 8.3 y $48.6 \%)(3,4)$ y estudios en del continente americano donde la prevalencia estuvo alrededor del $10.7 \%$ (3). La prevalencia de cetosis clínica $(0.6 \%)$ fue menor a la reportada por otros estudios de Colombia (6.0\%), (4), Holanda $(11.6 \%)(5)$, Canadá (3.7\%) (5) y México $(10.8 \%)(23)$. La prevalencia de cetosis subclínica también fue menor comparado a lo encontrado en el altiplano cundiboyacense de Colombia $(42.6 \%$ y $8 \%)(3,4)$, Inglaterra $(17 \%)$ (1) y Holanda $(47.2 \%)(2)$.

La explicación de estos resultados puede depender de la naturaleza heterogénea de las poblaciones y de la estabilidad de la oferta forrajera que ofrecen los sistemas productivos de pastoreo $(4,14)$ y estabulados (Total Mixed Rations - TMR), que pueden influir significativamente sobre la producción de leche y ácidos grasos volátiles cetogénicos (Butirato y acetato) o glucogénicos (Propionato) (17), producidos por las bacterias ruminales (24).

La evaluación de la condición corporal se ha usado principalmente como un indicador del balance energético (25), estos coinciden con nuestros resultados en que los animales con mayor CC (>3-3.5) tienen mayor riesgo de presentar cetosis. Según la definición de Gordon et al (10), estos casos pueden ser clasificados según su origen como Tipo 2, que ocurre principalmente durante periodos de disminución de consumo del parto o periparto, resultando en incremento de la actividad mitocondrial de la enzima CarnitilPalmitoil Transferasa I (CPT-1) (6). Lo anterior, sumado a las altas concentraciones de AGNE circulantes, en hembras obesas o de mayor condición corporal del hato pueden incrementar la posibilidad de producir cuerpos cetónicos excesivamente, aún en estados de hiperglicemia (17), dado que los AGNE también pueden ser re-esterificados a triglicéridos en el citosol del hepatocito y acumularse dentro y fuera de la célula por falta de transporte, ya que este depende de la síntesis y secreción de lipoproteína de muy baja densidad (VLDL). 
En rumiantes la producción de VLDL es baja, lo que representa, a su vez, más producción de cuerpos cetónicos e infiltración grasa del hígado particularmente en animales con una condición corporal alta, como ha sido observado en otros reportes en sistemas lecheros bajo condiciones de pastoreo donde el riesgo de cetosis ha sido mayor $(O R=5.25, p=0.018)$ tanto en Argentina (15) como en Colombia ( $R R=3.35, p=0.02)$ (4).

El número de partos ha sido un factor importante en la presentación de hipercetonemia y de cetosis bovina. El riesgo de hipercetonemia incrementó con el número de partos, así: animales con dos (OR: 3.1 , IC 95\% $(1.3-7.1) \mathrm{p}=0.009)$ y tres o más partos (OR: 3.7, IC $95(1.7-7.8) p=0.001)$ tuvieron mayor riesgo, respectivamente, en comparación con los animales de primer parto como lo han reportado otros estudio (2). esto puede estar asociado a que la producción de leche tiende a aumentar con el número de lactancias (25), lo cual incrementa los requerimientos energéticos generando cetosis primaria $(7,10)$.

De esta forma, la inducción de lipolisis ocasionada por las decrecientes concentraciones de glucosa al inicio de la lactancia o por la presentación de enfermedades concomitantes, pueden dar lugar al incremento excesivo de la concentración de Acetil-CoA en la mitocondria, generando la formación excesiva tanto de $\beta \mathrm{HB}$ como de Acetoacetato en circulación (6), por tanto puede ser fácilmente detectado en sangre, lo cual puede facilitar el diagnóstico.

De la misma forma, se ha documentado la relación existente entre la presentación de enfermedades puerperales y la cetosis bovina. Sin embargo, la prevalencia de fiebre de leche observada en este estudio (1.2\%) fue similar al 1.3 y $1.7 \%$ reportados en Canadá y Europa, respectivamente $(5,16)$ y menor al $3.3 \%$ encontrado en el altiplano cundiboyacense colombiano (4). Nuestros hallazgos coinciden con otros estudios en establecer un mayor riesgo de presentar cetosis en aquellos animales con casos clínicos de hipocalcemia (OR: 11.6, IC 95\% (3.79-35.40) $p=0.0001$. la disminución de consumo de alimento y anorexia que se observa durante la etapa 2 de los signos clínicos de la hipocalcemia explica en parte el incremento de los cuerpos cetónicos durante el curso de la enfermedad.

Otras enfermedades de ocurrencia común en el puerperio, como la retención de membranas fetales, estuvo asociada con la presentación de cetosis en este estudio (OR:7.9, IC 95\% (1.932.4) $p=0.004)$, al igual que ha sido reportada en otros estudios como un factor de riesgo para la cetosis de $(4,10)$, y cuya ocurrencia se ha explicado fisiológicamente por la disminución de consumo de alimento ( $2-6 \mathrm{Kg}$ /Día) que ocurre cuando se desarrollan signos clínicos de metritis (26). En este estudio ninguno de los animales diagnosticados con metritis tuvo historia de retención de placenta.

Vale la pena aclarar que la relación entre cetosis y la presentación de enfermedad puerperal (OR $=8.9$, IC (3.336-24.244) $p=0.0001)$, puede ser bidireccional y tener diversas causas por lo que atribuir una relación entre estas es difícil $(1,4)$. No obstante, se puede afirmar que la disminución en el consumo de alimento provocada por la presentación clínica de enfermedades puerperales (17), así como el balance energético negativo típico del postparto, afectan la concentración sanguínea de glucosa y desencadenan lipolisis severa y cetosis primaria hasta en un $30 \%$ de animales hipoglicémicos (27). Pero también, se ha reportado que la cetosis puede causar otras enfermedades posparto como metritis o claudicación $(18,19,28)$, debido al conocido compromiso de la función inmune de la vaca en transición y la resistencia a la insulina, generados por los altos niveles de AGNE circulantes (9).

La IL-6, TNF-a y proteínas de fase aguda (por ejemplo, Haptoglobina y amiloide sérico A) $(18,19)$, favorecen la presentación de estas entidades porque desencadenan alteraciones inflamatorias en el útero, favoreciendo infecciones secundarias (18). Por otra parte, la inducción de estos factores facilita el mecanismo de acción del ácido lipoteicoico y lipopolisacáridos producidos por bacterias ruminales, pudiendo causar inflamación o necrosis de la microvasculatura periférica y claudicación (19).

Aunque se ha observado que las concentraciones séricas de $\beta H B \geq 1.2$ (22) o $\geq 1.1 \mathrm{mmol} / \mathrm{Lt}$ (16) en sangre y $0.10 \mathrm{mmol} / \mathrm{Lt}$ en leche incrementan entre 4.7 (IC\%(2.06 - 10.91) $p=0.0003)-14.7$ (IC 95\% (7.2 - 29.8) $\mathrm{p}<0.01)$ veces el riesgo de manifestar signos clínicos de cetosis (16), en el presente estudio no se identificaron animales con signos clínicos de esta enfermedad.

La hipercetonemia se consideró como buen predictor de enfermedad puerperal aunque la probabilidad condicional de que un animal con un resultado positivo a la prueba esté enfermo y 
la proporción de animales enfermos y positivos sean bajas (Sen: 10.2\%; VPP: 59.4\%); En este estudio la hipercetonemia definida como la concentración sérica de $\beta \mathrm{HB}$ a un punto de corte de $1.2 \mathrm{mmol} / \mathrm{Lt}$ mostró ser eficiente en identificar individuos con enfermedad posparto con una Sensibilidad de $89.8 \%$ y especificidad del $93.4 \%$, sin embargo, no logra de manera acertada ser un buen método de diagnóstico para las enfermedades específicas del posparto evaluadas en el presente estudio ya que su sensibilidad varió entre el $0-50 \%$, pero su especificidad varió entre 91.8 - 92.6\% con lo cual el hallazgo de concentraciones de $\beta \mathrm{HB} \geq 1.2 \mathrm{mmol} /$ Lt permitió identificar adecuadamente a aquellos animales que no tenían ninguna condición clínica postparto (21). Esto adicionalmente se confirmó con los bajos valores positivos predictivos observados (0-59.4\%) y especialmente al observar el rango de valores predictivos negativos (95.6- 99.9\%), lo que indica claramente que la prueba es útil para descartar hipercetonemia en animales con otras condiciones clínicas (21).

De manera similar al observar los resultados de interpretación de la prueba utilizando el IP+, se pudo observar que la prueba podía ser eficiente en la identificación de animales que tuvieron retención de placenta (IP+ $=10.5)$ o enfermedad puerperal (IP+=13.6). En un estudio se observó que las concentraciones séricas $\beta \mathrm{HB}$ superiores a 1.0 y hasta $1.7 \mathrm{mmol} / \mathrm{Lt}$ estuvieron asociadas, pero no fueron predictores en los casos de desplazamiento de abomaso (IP $+=1.87-3.1$ ), cetosis clínica (IP+ $=2.17-3.8)$ o metritis (IP+ = 2.01 - 2.13) (5). En otro estudio el IP fue menor a 5 cuando se definió la prevalencia de cetosis clínica, metritis, desplazamiento de abomaso y claudicación (13). Esta interpretación posterior a la prueba implica que la definición del punto de corte para la interpretación del resultado de la prueba puede ser válido particularmente en la identificación de casos de enfermedad puerperal.

Una forma adicional de ver la eficiencia del punto de corte usado en este estudio $(\beta H B \geq 1.2$ $\mathrm{mmol} / \mathrm{Lt}$ ) y su relación con la identificación de patologías del posparto temprano, basado en el uso de curvas ROC, reveló que en el caso de hipercetonemia el área bajo la curva $(A B C=0.971$, $\mathrm{p}<0.05)$ fue altamente precisa para detectar casos de cetosis al mencionado punto de corte, esto es diferente a lo reportado por estudios en los que se observó una relación mucho menos precisa $(A B C=0.6-0.84)$ cuando el puntos de corte varió de 1.1-1.4 mmol/Lt $(13,14)$. La diferencia entre investigaciones ponen en manifiesto la importancia de la definición de un punto de corte adecuado a la hora de validar la prueba y resalta la importancia de considerar las diferencias inherentes al manejo de los sistemas de producción de leche $(3,20)$ el tipo de alimentación y su efecto sobre la producción de Ácidos Grasos Volátiles a nivel ruminal (24) en asocio con los factores que determinan el nivel de producción, la condición corporal al periparto (25) y los factores de riesgo que favorecen la presentación de enfermedades puerperales y el riesgo de cetosis $(3,4,14,15)$.

En conclusión, las concentraciones séricas de $\beta H B \geq 1.2 \mathrm{mmol} / \mathrm{Lt}$ sirven para alertar no solamente sobre la presentación de cetosis, sino de condiciones clínicas durante el puerperio, aunque, es un predictor específico de una enfermedad en particular, esto resulta útil en la detección temprana de condiciones que afectan la salud durante el postparto a nivel de hato. Adicionalmente, en condiciones de pastoreo la condición corporal alta, el número de partos la presentación de enfermedad puerperal, fiebre de leche o retención de placenta son factores de riesgo de hipercetonemia, lo cual resalta la necesidad implementar estrategias de monitoreo y prevención de estos factores en el control de esta entidad.

\section{Conflicto de intereses}

Los autores no declaran conflicto de intereses por la información presentada.

\section{Agradecimientos}

A la convocatoria 751 de 2016 de COLCIENCIAS y el grupo de investigación BIOGÉNESIS por financiar este trabajo. 


\section{REFERENCIAS}

1. Benedet A, Manuelian CL, Zidi A, Penasa M, De Marchi M. Invited review: $\beta$-hydroxybutyrate concentration in blood and milk and its associations with cow performance. Animal. 2019; 13(8):1676-1689. https://doi. org/10.1017/S175173111900034X

2. Vanholder T, Papen J, Bemers R, Vertenten $G$, Berge ACB. Risk factors for subclinical and clinical ketosis and association with production parameters in dairy cows in the Netherlands. J Dairy Sci. 2015; 98(2):880888. https://doi.org/10.3168/jds.2014-8362

3. Brunner N, Groeger S, Canelas Raposo J, Bruckmaier RM, Gross JJ. Prevalence of subclinical ketosis and production diseases in dairy cows in Central and South America, Africa, Asia, Australia, New Zealand, and Eastern Europe. Transl Anim Sci. 2019; 3(1):84-92. https://doi.org/10.1093/tas/txy102

4. Garzón-Audor A, Oliver-Espinosa O. Incidence and risk factors for ketosis in grazing dairy cattle in the Cundi-Boyacencian Andean plateau, Colombia. Trop Anim Health Prod. 2019; 51(6):1481-1487. https://doi. org/10.1007/s11250-019-01835-z

5. Seifi HA, LeBlanc SJ, Leslie KE, Duffield TF. Metabolic predictors of post-partum disease and culling risk in dairy cattle. Vet J. $2011 ; 188(2): 216-220$. https://doi. org/10.1016/j.tvjl.2010.04.007

6. Herdt TH. Ruminant Adaptation to Negative Energy Balance. Vet Clin North Am Food Anim Pract. $2000 ; 16(2): 215-230$. https:// doi.org/10.1016/s0749-0720(15)30102-x

7. Garzon A., Oliver OJ. Epidemiología de la cetosis en bovinos: una revisión. Rev CES. 2018; 13(1):43-61. https://doi. org/10.21615/cesmvz.13.1.4

8. Holtenius $\mathrm{P}$, Holtenius K. New aspects of ketone bodies in energy metabolism of dairy cows: a review. Zentralbl Veterinarmed A. $1996 ; 43(10): 579-587$. https://doi. org/10.1111/j.1439-0442.1996.tb00491.x
9. Contreras GA, Sordillo LM. Lipid mobilization and inflammatory responses during the transition period of dairy cows. Comp Immunol Microbiol Infect Dis. 2011; 34(3):281-289. https://doi.org/10.1016/j. cimid.2011.01.004

10. Gordon JL, LeBlanc SJ, Duffield TF. Ketosis treatment in lactating dairy cattle. Vet Clin North Am - Food Anim Pract. 2013; 29(2):433-445. https://doi.org/10.1016/j. cvfa.2013.03.001

11. McArt JAA, Nydam D V., Oetzel GR, Overton $T R$, Ospina PA. Elevated non-esterified fatty acids and $\beta$-hydroxybutyrate and their association with transition dairy cow performance. Vet J. 2013; 198(3):560-570. https://doi.org/10.1016/j.tvjl.2013.08.011

12. Tatone EH, Duffield TF, LeBlanc SJ, DeVries $\mathrm{TJ}$, Gordon JL. Investigating the within-herd prevalence and risk factors for ketosis in dairy cattle in Ontario as diagnosed by the test-day concentration of $\beta$-hydroxybutyrate in milk. J Dairy Sci. 2017; 100(2):13081318. https://doi.org/10.3168/jds.2016$\underline{11453}$

13. Suthar VS, Canelas-Raposo J, Deniz A, Heuwieser W. Prevalence of subclinical ketosis and relationships with postpartum diseases in European dairy cows. J Dairy Sci. 2013; 96(5):2925-2938. https://doi. org/10.3168/jds.2012-6035

14. Compton CWR, Young L, McDougall S. Subclinical ketosis in post-partum dairy cows fed a predominantly pasture-based diet: defining cut-points for diagnosis using concentrations of beta-hydroxybutyrate in blood and determining prevalence. $\mathrm{N} Z$ Vet J. 2015; 63(5):241-248. https://doi.org/1 $\underline{0.1080 / 00480169.2014 .999841}$

15. Garro CJ, Mian L, Cobos Roldán M. Subclinical ketosis in dairy cows: Prevalence and risk factors in grazing production system. J Anim Physiol Anim Nutr (Berl). 2014; 98(5):838844. https://doi.org/10.1111/jpn.12141 
16. Berge AC, Vertenten G. A field study to determine the prevalence, dairy herd management systems, and fresh cow clinical conditions associated with ketosis in western European dairy herds. J Dairy Sci. 2014; 97(4):2145-2154. https://doi.org/10.3168/ jds.2013-7163

17. McFarlane D, Fleming SA. Large Animal Internal Medicine. 5th ed. Chapter 41, Endocrine and Metabolic Diseases. Estados Unidos: Mosby; 2014. https://www.elsevier. com/books/large-animal-internal-medicine/ smith/978-0-323-08839-8

18. Dervishi E, Zhang G, Hailemariam D, Goldansaz SA, Deng Q, Dunn SM, et al. Alterations in innate immunity reactants and carbohydrate and lipid metabolism precede occurrence of metritis in transition dairy cows. Res Vet Sci. 2016; 104(1):30-39. https://doi.org/10.1016/j.rvsc.2015.11.004

19. Zhang G, Hailemariam D, Dervishi E, Deng Q, Goldansaz SA, Dunn SM, et al. Alterations of innate immunity reactants in transition dairy cows before clinical signs of lameness. Animals. 2015; 5(3):717-747. https://doi. org/10.3390/ani5030381

20. Tatone EH, Gordon JL, Hubbs J, LeBlanc SJ, DeVries TJ, Duffield TF. A systematic review and meta-analysis of the diagnostic accuracy of point-of-care tests for the detection of hyperketonemia in dairy cows. Prev Vet Med. 2016; 130(1):18-32. https:// doi.org/10.1016/j.prevetmed.2016.06.002

21. Erb HN. Prior probability (the pretest best guess) affects predictive values of diagnostic tests. Vet Clin Pathol. 2011; 40(2):154158. https://doi.org/10.1111/j.1939165X.2011.00315.X

22. McGee S. Simplifying likelihood ratios. J Gen Intern Med. 2002; 17(8):647650. https://doi.org/10.1046/j.15251497.2002.10750.x
23. Mellado M, Dávila A, Gaytán L, Macías-Cruz $U$, Avendaño-Reyes L, García E. Risk factors for clinical ketosis and association with milk production and reproduction variables in dairy cows in a hot environment. Trop Anim Health Prod. 2018; 50(7):1611-1616. https://doi.org/10.1007/s11250-0181602-y

24. Miettinen $\mathrm{H}$, Huhtanen P. Effects of the ratio of ruminal propionate to butyrate on milk yield and blood metabolites in dairy cows. J Dairy Sci. 2010; 79(5):851-861. https://doi. org/10.3168/jds.s0022-0302(96)76434-2

25. Rathbun FM, Pralle RS, Bertics SJ, Armentano LE, Cho K, Do C, et al. Relationships between body condition score change, prior midlactation phenotypic residual feed intake, and hyperketonemia onset in transition dairy cows. J Dairy Sci. 2017; 100(5):3685-3696. https://doi.org/10.3168/jds.2016-12085

26. Huzzey JM, Veira DM, Weary DM, von Keyserlingk MAG. prepartum behavior and dry matter intake identify dairy cows at risk for metritis. J Dairy Sci. 2007; 90(7):32203233. https://doi.org/10.3168/jds.2006$\underline{807}$

27. Dubuc J, Buczinski S. Short communication: Cow- and herd-level prevalence of hypoglycemia in hyperketonemic postpartum dairy cows. J Dairy Sci. 2018; 101(4):33743379. https://doi.org/10.3168/jds.2017$\underline{13773}$

28. Suthar, V.S., Canelas-Raposo, J., Deniz, A., Heuwieser, W. Prevalence of subclinical ketosis and relationships with postpartum diseases in European dairy cows. J. Dairy Sci. 2013; 96(5):1-14. https://doi.org/10.3168/ jds.2012-6035 